\title{
EXPONENTIAL INEQUALITIES AND COMPLETE CONVERGENCE OF EXTENDED ACCEPTABLE RANDOM VARIABLES $^{\dagger}$
}

\author{
JEONG-YEOL CHOI AND JONG-IL BAEK*
}

\begin{abstract}
Giuliano Antonini et al.(2008) have introduced the concept of extended acceptability and the results show that the extended acceptability structure has no effect on the exponential inequality except replacing a constant $M=1$ with a constant $M>0$. We discuss the complete convergence for extended acceptable random variables by using the exponential inequality.
\end{abstract}

AMS Mathematics Subject Classification : 60F15.

Key words and phrases : Acceptable, Extended negatively orthant dependence, Extended acceptable random variable, Exponential inequality, Complete convergence.

\section{Introduction}

Giuliano Antonini et al.(2008) recently have introduced the concept of acceptability as follows; A finite sequence $\left\{X_{i}, 1 \leq i \leq n\right\}$ of random variables is said to be acceptable if for any real $\lambda$,

$$
E \exp \left(\lambda \sum_{i=1}^{n} X_{i}\right) \leq \Pi_{i=1}^{n} E \exp \left(\lambda X_{i}\right) .
$$

An infinite sequence $\left\{X_{n}, n \geq 1\right\}$ of random variable is acceptable if every finite subcollection is acceptable. They also mentioned that a sequence of negatively dependent random variables with a finite Laplace transform or finite moment generating function near zero provides us an example of acceptable random variables. In addition, Liu(2009) introduced the concept of extended negative orthant dependence by extending the negative orthant dependence as follows;

Received November 28, 2012. Revised February 25, 2013. Accepted March 4, 2013. ${ }^{*}$ Corresponding author. ${ }^{\dagger}$ This paper was supported by WonKwang University Research Grant in 2012 .

(c) 2013 Korean SIGCAM and KSCAM. 
A sequence of random variables $\left\{X_{i}, i \geq 1\right\}$ is said to be extended negatively orthant dependent $(E N O D)$ if there exists a constant $M>0$ such that both

$$
P\left(X_{1} \leq x_{1}, \cdots, X_{n} \leq x_{n}\right) \leq M \Pi_{i=1}^{n} P\left(X_{i} \leq x_{i}\right)
$$

and

$$
P\left(X_{1}>x_{1}, \cdots, X_{n}>x_{n}\right) \leq M \Pi_{i=1}^{n} P\left(X_{i}>x_{i}\right)
$$

hold for each $n=1,2, \cdots$ and all $x_{1}, \cdots, x_{n}$. Recall that the sequence $\left\{X_{i}, i \geq\right.$ $1\}$ is said to be negatively orthant dependent $(N O D)$ if both (1.2) and (1.3) hold when $M=1$; it is called positively orthant dependent $(P O D)$ if $(1.2)$ and (1.3) hold both in the reverse direction when $M=1$. Obviously, NOD (See JoagDev and Proschan(1983) and Baek et al.(2011)) sequence must be an ENOD sequence.

We defined an extended acceptability from the definitions of acceptability and extended orthant dependence as follows.

Definition 1.1. A finite sequence $\left\{X_{i}, 1 \leq i \leq n\right\}$ of random variables is said to be extended acceptable if there exists a constant $M>0$ such that for any real $\lambda$

$$
E \exp \left(\lambda \sum_{i=1}^{n} X_{i}\right) \leq M \Pi_{i=1}^{n} E \exp \left(\lambda X_{i}\right) .
$$

An infinite sequence $\left\{X_{n}, n \geq 1\right\}$ of random variables is extended acceptable if every finite subcollection is extended acceptable. A sequence $\left\{X_{i}, i \geq 1\right\}$ of random variables is obviously acceptable if (1.4) holds when $M=1$ and hence an acceptable sequence must be an extended acceptable sequence. In addition, (1.2) and (1.3) obviously satisfy (1.4). Therefore, the ENOD random variables are extended acceptable random variables.

From the similar method in Giuliano Antonini etc., a sequence of ENOD random variables with a finite Laplace transform or finite moment generating function near zero provides us an example of extended acceptable random variables. In particular, there have been many investigations on the exponential inequality for dependent random variables. For examples, Kim et al.(2007) and Xing et al.(2010),and Wang et al.(2010) had established an exponential inequality for dependent random variables. Sung et al.(2011) obtained an exponential inequality for identically distributed acceptable random variables as follows.

Theorem 1.2. Let $\left\{X_{n}, n \geq 1\right\}$ be a sequence of identically distributed acceptable random variables with Ee $e^{\delta\left|X_{1}\right|}<\infty$ for some $\delta>0$. Then for any $0<\epsilon \leq K \delta$,

$$
P\left(\left|S_{n}-E S_{n}\right| \geq n \epsilon\right) \leq 2 \exp \left(-\frac{n \epsilon^{2}}{4 K}\right),
$$

where $S_{n}=X_{1}+\cdots+X_{n}$. 
The main goal of our paper is to extend Theorem 1.1 to extended acceptable random variables; discuss the above result for extended acceptable random variables and in addition complete convergence of extended acceptable. This paper is organized as follows. In section 2 , we provide the establish the exponential inequalities for sum of extended acceptable random variables and in section 3 , we obtain a result dealing with the complete convergence for these random variables by using the exponential inequality.

\section{Exponential inequalities for extended acceptable random variables}

First we extend Sung et al. s'(2011) results on acceptable structure to the extended acceptability cases.

Theorem 2.1. Let $\left\{X_{n}, n \geq 1\right\}$ be a sequence of identically distributed and extended acceptable random variables with $E e^{\delta\left|X_{1}\right|}<\infty$ for some $\delta>0$. Then there exists a constant $M>0$ such that for any $0<\epsilon \leq K \delta$

$$
P\left(S_{n}-E S_{n} \geq n \epsilon\right) \leq M \exp \left(-\frac{n \epsilon^{2}}{4 K}\right)
$$

and

$$
P\left(\left|S_{n}-E S_{n}\right| \geq n \epsilon\right) \leq 2 M \exp \left(-\frac{n \epsilon^{2}}{4 K}\right)
$$

where $S_{n}=X_{1}+\cdots+X_{n}$.

Proof. The proof is similar to that of Theorem 2.1 in Sung et al.(2011). Suppose that $0<\epsilon \leq K \delta$, then by Markov's inequality, the definition of extended acceptable random variables and Sung et al's Lemma 2.1, for any $0<\lambda \leq \delta / 2$,

$$
\begin{aligned}
& P\left(\sum_{i=1}^{n}\left(X_{i}-E X_{i}\right)>n \epsilon\right)=P\left(\exp \left(\lambda \sum_{i=1}^{n}\left(X_{i}-E X_{i}\right)\right)>\exp (\lambda n \epsilon)\right) \\
\leq & \exp (-\lambda n \epsilon) E \exp \left(\lambda \sum_{i=1}^{n}\left(X_{i}-E X_{i}\right)\right) \\
\leq & M \exp (-\lambda n \epsilon) \prod_{i=1}^{n} \exp \left(\lambda\left(X_{i}-E X_{i}\right)\right) \\
\leq & M \exp (-\lambda n \epsilon) \prod_{i=1}^{n} \exp \left(K \lambda^{2}\right)=M \exp \left(-\lambda n \epsilon+K \lambda^{2} n\right) .
\end{aligned}
$$

We take $\lambda=\epsilon /(2 K)$ in the last term and note that $\epsilon /(2 K) \leq \delta / 2$ by condition $0<\epsilon \leq K \delta$. Thus, we get that

$$
P\left(\sum_{i=1}^{n}\left(X_{i}-E X_{i}\right)>n \epsilon\right) \leq M \exp \left(-\frac{n \epsilon^{2}}{4 K}\right) .
$$


Since a sequence $\left\{-X_{n}, n \geq 1\right\}$ is also extended acceptable, by replacing $X_{i}$ with $-X_{i}$ in the above statement, we obtain that

$$
P\left(-\sum_{i=1}^{n}\left(X_{i}-E X_{i}\right)>n \epsilon\right) \leq M \exp \left(-\frac{n \epsilon^{2}}{4 K}\right) .
$$

It follows from (2.4) and (2.5) that

$$
\begin{aligned}
& P\left(\left|\sum_{i=1}^{n}\left(X_{i}-E X_{i}\right)\right|>n \epsilon\right) \\
= & P\left(\sum_{i=1}^{n}\left(X_{i}-E X_{i}\right)>n \epsilon\right)+P\left(-\sum_{i=1}^{n}\left(X_{i}-E X_{i}\right)>n \epsilon\right) \leq 2 M \exp \left(-\frac{n \epsilon^{2}}{4 K}\right) .
\end{aligned}
$$

Remark 2.1. Theorem 2.1 of Sung et al.(2011) is a special case of Theorem 2.2 when $M=1$.

From Theorem 2.1, we can get the result of Corollary 2.2 as follows.

Corollary 2.2. Let $\left\{X_{n}, n \geq 1\right\}$ be a sequence of identically distributed and extended acceptable random variables with $E e^{\delta\left|X_{1}\right|}<\infty$ for some $\delta>0$. Set $\epsilon_{n}=2(K \alpha(\log n) / n)^{1 / 2}$, where $\alpha>0$ and $K=2\left(E\left|X_{1}\right|^{4}\right)^{1 / 2} E\left(e^{\delta\left|X_{1}\right|}\right)$. Then there exists a constant $M>0$ such that for $\alpha>1$

$$
\left.\sum_{n=1}^{\infty} P\left|S_{n}-E S_{n}\right|>n \epsilon_{n}\right) \leq 2 M \sum_{n=1}^{\infty} \exp (-\alpha \log n)<\infty .
$$

Proof. Let $\epsilon_{n}=2(K \alpha(\log n) / n)^{1 / 2}$, where $\alpha>1$ and $K=2\left(E\left|X_{1}\right|^{4}\right)^{1 / 2} E\left(e^{\delta\left|X_{1}\right|}\right)$. Then $\epsilon_{n} /(K \delta) \leq 1$ for all large $n$. Hence, the result follows directly from Theorem 2.1 .

Theorem 2.2. Let $\left\{X_{n}, n \geq 1\right\}$ be a sequence of extended acceptable random variables with $E e^{\delta\left|X_{i}\right|}<\infty$ for some $\delta>0$ and for each $i \geq 1$ and $\left\{g_{n}, n \geq 1\right\}$ be a sequence of positive numbers with $G_{n}=\sum_{i=1}^{n} g_{i}$ for each $n \geq 1$. For fixed $n \geq 1$, if there exists a positive number $T$ such that

$$
E \exp \left(t X_{i}\right) \leq \exp \left(\frac{1}{2} g_{i} t^{2}\right), 0 \leq t \leq T, i=1,2, \cdots, n,
$$

then, for some constant $M>0$

$$
P\left(S_{n} \geq x\right) \leq\left\{\begin{array}{l}
M \exp \left(-\frac{x^{2}}{2 G_{n}}\right), 0 \leq x<G_{n} T \\
M \exp \left(-\frac{T x}{2}\right), x \geq G_{n} T
\end{array}\right.
$$

Proof. For each $x$, by Markov's inequality we see that

$$
P\left(S_{n} \geq x\right) \leq \exp (-t x) E \exp \left(t S_{n}\right), t>0 .
$$


By (1.4) there exists a constant $M>0$ such that for $0<t \leq T$

$$
E \exp \left(t S_{n}\right)=E\left(\prod_{i=1}^{n} \exp \left(t X_{i}\right)\right) \leq M \prod_{i=1}^{n} E \exp \left(t X_{i}\right) \leq M \exp \left(\frac{G_{n} t^{2}}{2}\right) .
$$

It follows from (2.8) and (2.9) that

$$
P\left(S_{n} \geq x\right) \leq M \inf _{0<t \leq T} \exp \left(\frac{G_{n} t^{2}}{2}-t x\right)=M \exp \left(\inf _{0<t \leq T}\left(\frac{G_{n} t^{2}}{2}-t x\right)\right) .
$$

For fixed $x \geq 0$, if $T>\frac{x}{G_{n}} \geq 0$, then

$$
\exp \left(\inf _{0<t \leq T}\left(\frac{G_{n} t^{2}}{2}-t x\right)\right)=\exp \left(-\frac{x^{2}}{2 G_{n}}\right)
$$

and if $T<\frac{x}{G_{n}}$, then

$$
\exp \left(\inf _{0<t \leq T}\left(\frac{G_{n} t^{2}}{2}-t x\right)\right)=\exp \left(\frac{G_{n} t^{2}}{2}-T x\right) \leq \exp \left(-\frac{T x}{2}\right) .
$$

From (2.10)-(2.12), we obtain the result of (2.7).

Theorem 2.3. Let $\left\{X_{n}, n \geq 1\right\}$ be a sequence of extended acceptable random variables with $E X_{i}=0, E e^{\bar{\delta}\left|X_{i}\right|}<\infty$ for some $\delta>0$ and $\left|X_{i}\right| \leq b$ for each $i \geq 1$, where $b$ is a positive constant. Denote $B_{n}^{2}=\sum_{i=1}^{n} E X_{i}^{2}$ for each $n \geq 1$. Then, for any $\epsilon>0$ and for some constant $M>0$

and

$$
P\left(S_{n} \geq \epsilon\right) \leq M \exp \left\{-\frac{\epsilon^{2}}{2\left(2 B_{n}^{2}+b \epsilon\right)}\right\}
$$

$$
P\left(\left|S_{n}\right| \geq \epsilon\right) \leq 2 M \exp \left\{-\frac{\epsilon^{2}}{2\left(2 B_{n}^{2}+b \epsilon\right)}\right\}
$$

Proof. Clearly, for any $0<t \leq \frac{1}{b}\left|t X_{i}\right| \leq 1$ by assumption $\left|X_{i}\right| \leq b$. Thus, by the fact that $e^{y}>1+y, y>0$ we obtain

$$
E \exp \left|t X_{i}\right|=1+\sum_{n=2}^{\infty} \frac{E\left(t X_{i}\right)^{n}}{n !} \leq 1+t^{2} E X_{i}^{2} \leq \exp \left(t^{2} E X_{i}^{2}\right)
$$

By Markov's inequality, Definition 1.1 and (2.15)

$$
\begin{gathered}
P\left(S_{n} \geq \epsilon\right) \leq \exp (-t \epsilon) E \exp \left(t S_{n}\right) \\
\leq M e^{-t \epsilon} \prod_{i=1}^{n} E \exp \left(t X_{i}\right) \leq M \exp \left(-t \epsilon+t^{2} B_{n}^{2}\right),
\end{gathered}
$$

which yields (2.13) by taking $t=\epsilon /\left(2 B_{n}^{2}+b \epsilon\right)$. Since $\left\{-X_{n}, n \geq 1\right\}$ is also a sequence of extended acceptable random variables, it follows from (2.13) that

$$
P\left(S_{n} \leq-\epsilon\right)=P\left(-S_{n} \geq \epsilon\right) \leq M \exp \left\{-\frac{\epsilon^{2}}{2\left(2 B_{n}^{2}+b \epsilon\right)}\right\} .
$$

Combining (2.13) and (2.16) yields (2.14). 
Theorem 2.4. Let $\left\{X_{n}, n \geq 1\right\}$ be a sequence of extended acceptable random variables. If there exist sequences of real numbers $\left\{a_{n}, n \geq 1\right\}$ and $\left\{b_{n}, n \geq 1\right\}$ such that $a_{i} \leq X_{i} \leq b_{i}$ for each $i \geq 1$. Then, for any $\epsilon>0$ and some constant $M>0$

$$
P\left(S_{n}-E S_{n} \geq n \epsilon\right) \leq M \exp \left\{-\frac{2 n^{2} \epsilon^{2}}{\sum_{i=1}^{n}\left(b_{i}-a_{i}\right)^{2}}\right\}
$$

and

$$
P\left(\left|S_{n}-E S_{n}\right| \geq n \epsilon\right) \leq 2 M \exp \left\{-\frac{2 n^{2} \epsilon^{2}}{\sum_{i=1}^{n}\left(b_{i}-a_{i}\right)^{2}}\right\}, n \geq 1
$$

Proof. For any $h>0$ by Markov's inequality, we have

$$
P\left(S_{n}-E S_{n} \geq n \epsilon\right) \leq E \exp \left[h\left(S_{n}-E S_{n}-n \epsilon\right)\right]
$$

It follows from Definition 1.1 that for some constant $M>0$

$$
\begin{aligned}
E \exp \left[h\left(S_{n}-E S_{n}-n \epsilon\right)\right] & =\exp (-h n \epsilon) E \prod_{i=1}^{n} \exp \left[h\left(X_{i}-E X_{i}\right)\right] \\
& \leq M \exp (-h n \epsilon) \prod_{i=1}^{n} \exp \left[h\left(X_{i}-E X_{i}\right)\right]
\end{aligned}
$$

Hoeffding(1963) proved that if $a \leq X \leq b$, then for any $h>0$

$$
E \exp [h(X-E X)] \leq \exp \left[h^{2}(b-a)^{2} / 8\right] .
$$

By $(2.20)$ and $(2.21)$

$$
P\left(S_{n}-E S_{n} \geq n \epsilon\right) \leq M \exp \left(-h n \epsilon+\frac{1}{8} h^{2} \sum_{i=1}^{n}\left(b_{i}-a_{i}\right)^{2}\right)
$$

It is easily seen that the right hand side of (2.22) has its minimum at $h=$ $\frac{4 n \epsilon}{\sum_{i=1}^{n}\left(b_{i}-a_{i}\right)^{2}}$. Inserting this value in $(2.22)$ we obtain (2.17). Since $\left\{-X_{n}\right\}$ is also a sequence of extended acceptable random variables by (2.17) we obtain

$$
P\left(S_{n}-E S_{n} \leq-n \epsilon\right) \leq M \exp \left\{-\frac{2 n^{2} \epsilon^{2}}{\sum_{i=1}^{n}\left(b_{i}-a_{i}\right)^{2}}\right\}, n \geq 1 .
$$

From (2.17) and (2.21), we obtain the result of (2.18).

\section{Complete convergence for extended acceptable random variables}

Theorem 3.1. Let $\left\{X_{n}, n \geq 1\right\}$ be a sequence of identically distributed and extended acceptable random variables with $E X_{1}=0$ and $E e^{\delta\left|X_{1}\right|}<\infty$ for some $\delta>0$. Then $n^{-1}\left(S_{n}-E S_{n}\right) \rightarrow 0$ completely as $n \rightarrow \infty$.

Proof. By using Theorem 2.1, we can be obtained the result of Theorem 3.1 and the proof is omitted. 
Theorem 3.2. Let $\left\{X_{n}, n \geq 1\right\}$ be a sequence of extended acceptable random variables with $E X_{i}=0, E e^{\delta\left|X_{i}\right|}<\infty$ for some $\delta>0$ and $\left|X_{i}\right| \leq b$ for each $i \geq 1$, where $b$ is a positive constant. If $\sum_{i=1}^{\infty} E X_{i}^{2}<\infty$ then for any $r>0$

$$
n^{-r} S_{n} \rightarrow 0 \text { completely as } n \rightarrow \infty \text {. }
$$

Proof. It follows from (2.14) that for any $\epsilon>0$ and some constant $M>0$

$$
\begin{aligned}
\sum_{n=1}^{\infty} P\left(\left|S_{n}\right| \geq n^{r} \epsilon\right) & \leq 2 M \sum_{n=1}^{\infty} \exp \left\{-\frac{n^{2 r} \epsilon^{2}}{2\left(2 \sum_{i=1}^{n} E X_{i}^{2}+b n^{r} \epsilon\right)}\right\} \\
& \leq 2 \sum_{n=1}^{\infty}[\exp (-C)]^{n^{r}}<\infty
\end{aligned}
$$

which yields (3.1), where $C$ is a positive number not depending on $n$.

Theorem 3.3. Let $\left\{X_{n}, n \geq 1\right\}$ be a sequence of extended acceptable random variables with $E e^{\delta\left|X_{i}\right|}<\infty$ for some $\delta>0$ and $\left|X_{i}\right| \leq C<\infty$ for each $i \geq 1$, where $C$ is a positive constant. Then, for any $r>\frac{1}{2}$

$$
n^{-r}\left(S_{n}-E S_{n}\right) \rightarrow 0 \text { completely as } n \rightarrow \infty .
$$

Proof. For any $\epsilon>0$ and some constant $M>0$ we obtain

$$
\sum_{n=1}^{\infty} P\left(\left|S_{n}-E S_{n}\right| \geq n^{r} \epsilon\right) \leq 2 M \sum_{n=1}^{\infty}\left[\exp \left(-\frac{\epsilon^{2}}{2 C^{2}}\right)\right]^{n^{2 r-1}}<\infty
$$

by Theorem 2.4. Hence, $n^{-r}\left(S_{n}-E S_{n}\right) \rightarrow 0$ completely as $n \rightarrow \infty$.

\section{REFERENCES}

1. E. L. Lehmann, Some concepts of dependence, Ann. Math. Statist. 37 (1966), 1137-1153.

2. G. Xing and S. Yang, An exponential inequality for strictly stationary negatively associated random variables, Commun. Stat. Theor. Meth. 39 (2010), 340-349.

3. J.I. Baek and H.Y. Seo, On the convergence for ND random variables with applications, JAMI. 29 (2011), 1351-1361.

4. K. Joag-Dev and F. Proschan, Negative association of random variables with applications, Ann. Statist. 11 (1983), 286-295.

5. L. Liu, Pricise large deviations for dependent random variables with heavy tails, Statist. Probab. Lett. 99 (2009), 1290-1298.

6. R. Giuliano Antonini, Y. Kozachenko and A. Volodin, Convergence of series dependent $\varphi$-sub Gaussian random variables, J. Math. Anal. Appl. 338 (2008), 1188-1203.

7. S. Sung, P. Srisuradetchai and A. Volodin, A note on the exponential inequality for a class of dependent random variables, J. Korean Stat. Soc. 40 (2011) 109-114.

8. T. S. Kim and H. C. Kim, On the exponential inequality for negatively dependent sequence, Commun. Korean Soc. 22 (2007), 315-321.

9. W. Hoeffding, Probability inequalities for some of bounded random variables, J. Amer.Statist. Assoc. 58 (1963), 13-30. 
10. X. Wang, S. Hu, W. Yang and N. Ling, Exponential inequalities and inverse moment for NOD sequence, Statist. Probab. Lett. 80 (2010), 452-461.

Choi Jeong Yeol is working at School of Mathematrics and Informational statistics in Wonkwang University. His research interest in Analysis and probability theory.

School of mathematics and Informational statistics and Institute of Basic Natural Science, Wonkwang University, Iksan 570-749, Korea.

e-mail: jychoi@wku.ac.kr

Baek Jong Il is working at School of Mathematrics and Informational statistics and Institute of Basic Natural Science in Wonkwang University. His research interests focus on the probability theory, time series and reliability theory.

School of mathematics and Informational statistics and Institute of Basic Natural Science, Wonkwang University, Iksan 570-749, Korea.

e-mail: jibaek@wku.ac.kr 